\title{
Association Between Single-Nucleotide Polymorphisms in Breast Cancer Susceptibility Genes and Clinicopathological Characteristics
}

This article was published in the following Dove Press journal: Clinical Epidemiology

\author{
Shouman Wang ${ }^{1,2}$ \\ Kejing Zhang ${ }^{1,2}$ \\ Lili Tang ${ }^{1,2}$ \\ Yuan Yang ${ }^{1,2}$ \\ Hao Wang ${ }^{3}$ \\ Zhiyang Zhou ${ }^{1,2}$ \\ Jian Pang 1,2 \\ Feiyu Chen ${ }^{1,2}$
}

'Department of Breast Surgery, Xiangya Hospital, Central South University, Changsha, Hunan Province, People's Republic of China; ${ }^{2}$ Clinical Research Center for Breast Cancer in Hunan Province, Changsha, Hunan Province, People's Republic of China; ${ }^{3}$ Department of Breast Surgery, Second People's Hospital of Sichuan Province, Chengdu, Sichuan Province, People's Republic of China
Correspondence: Feiyu Chen

Department of Breast Surgery, Xiangya Hospital, Central South University, No. 87 Xiangya Road, Changsha City, Hunan Province, 410008, People's Republic of China

Tel +86 I34675II 343

Fax +86073189753615

Email chenfy13@csu.edu.cn
Objective: The purpose of the present study was to evaluate the associations between seven tagging single nucleotide polymorphisms (tSNPs) and risk of breast cancer assessed by tumor pathological characteristics and body mass index (BMI).

Methods: Seven tSNPs of four breast cancer susceptibility genes were analyzed in 734 Chinese women with breast cancer and 672 age-matched healthy controls; then, the association with clinicopathological characteristics, BMI, molecular subtype, TNM ( $\mathrm{T}$, tumor; N, lymph node; $\mathrm{M}$, metastasis) staging and lymph node status was determined by unconditional logistic regression.

Results: Rs12951053 in TP53 and rs16945628 in BRIP1, displayed increased risk of breast cancer in the $\mathrm{BMI} \geqq 25 \mathrm{~kg} / \mathrm{m}^{2}$ group $(\mathrm{OR}=1.50,95 \% \mathrm{CI}: 1.02-2.21, \mathrm{P}=0.041$ and $\mathrm{OR}=1.92$, 95\% CI: $1.13-3.26, \mathrm{P}=0.015$, respectively). The other five tSNPs (rs 1805812 , rs 2735385 and rs6999227 in NBS1, rs7220719 in BRIP1 and rs2299941 in PTEN) displayed a decreased risk of breast cancer in the $18.5 \leq \mathrm{BMI}<25 \mathrm{~kg} / \mathrm{m}^{2}$ group. Rs12951053 in TP53 and rs7220719 in BRIP1 exhibited an increased risk of triple-negative breast cancer $(\mathrm{OR}=1.50,95 \% \mathrm{CI}$ : 1.05-2.15, $\mathrm{P}=0.026$ and $\mathrm{OR}=2.13,95 \% \mathrm{CI}: 1.05-4.29, \mathrm{P}=0.032$, respectively), but three tSNPs in NBS1 (rs1805812, rs2735385 and rs6999227) all displayed a negative association with both luminal B and triple-negative breast cancer. The tSNP rs2299941 in PTEN also exhibited a negative association with each molecular subtype, except triple-negative breast cancer. The majority of tSNPs displayed a negative association with stage II or III breast cancer. Most tSNPs showed a negative association with breast cancer that was lymph node negative or with 1-3 positive nodes. Only rs12951053 in TP53 displayed a positive association with lymph node-negative breast cancer ( $\mathrm{OR}=1.43,95 \% \mathrm{CI}: 1.08-1.91, \mathrm{P}=0.013)$.

Conclusion: The majority of tSNPs displayed a negative association with breast cancer and only a few tSNPs (rs12951053 in TP53, rs16945628 and rs7220719 in BRIP1) showed an increased risk of breast cancer as defined by clinicopathological characteristics.

Keywords: breast cancer, SNP, molecular subtype, BRIP1, NBS1

According to the latest statistics, among all malignant tumors in women, the incidence rate of breast cancer ranks highest in both China and the United States. ${ }^{1,2}$ The mortality rate ranks second for all female malignant tumors in the United States, with a declining trend year-by-year, attributed to the early detection of breast cancer and individualized treatment according to the molecular classification of the cancer. With the development of breast cancer susceptibility gene panels, evidence that genetic factors can also influence tumor subtype is provided by the fact that patients with triple-negative breast cancer (TNBC) tend to more frequently 
have mutations in the BRCA1 (8.5\%) and BRCA2 (2.7\%) genes, in addition to other low-to-medium penetrance genes, including PALB2 (1.2\%) and BARD1, RAD51D, RAD51C, and BRIP1 $(0.3 \%$ to $0.5 \%){ }^{3}$ Furthermore, in patients with HER2 (human epidermal growth factor receptor-2)-negative metastatic breast cancer and a germline BRCA mutation, monotherapy with the PARP (poly ADP-ribose polymerase) inhibitor olaparib provides a significant benefit over standard therapy, especially in TNBC (OR $=0.43,95 \%$ CI: $0.29-0.063) .{ }^{4}$ The majority of previous reports suggest that genetic polymorphisms possibly influence the pathological subtype of breast cancer in only ER (estrogen receptor)-positive or ER-negative breast cancer. $^{5-7}$ There are only a few published reports of risk factors for combined ER, PR (progesterone receptor) and HER2 status, one of which is a study by O'Brien et al that demonstrates that several SNPs in TNRC9/TOX3 are associated with luminal A or basal-like breast cancer, and one SNP (rs3104746) that is associated with both. ${ }^{8}$ Therefore, understanding the associations between genetic polymorphisms and clinicopathological features of breast cancer may ultimately result in improvements in prevention, early detection, and treatment. In a previous study, seven tagging SNPs (tSNPs) of four genes were found to be significantly associated with breast cancer risk under a codominant model in unselected cases, including rs12951053 located in TP53, rs1805812, rs2735385 and rs6999227 in NBS1, rs16945628 and rs7220719 in BRIP1, and rs2299941 in PTEN. ${ }^{9}$ Therefore, the purpose of the present study was to evaluate the associations between these tSNPs and breast cancer risk as defined by tumor pathological characteristics and body mass index (BMI).

\section{Materials and Methods}

\section{Subjects}

As we have described in a previous study, ${ }^{9,10} 734$ female patients with pathologically confirmed breast cancer were recruited from the Department of Breast Surgery of Central South University's Xiangya Hospital, Changsha, between January 2007 and October 2011, and the Department of Breast Surgery of the Second People's Hospital of Sichuan Province, Chengdu, China, between November 2010 and May 2011. In addition, a total of 672 aged-matched women with no personal or family history of cancer were enrolled as normal controls at the Health Management Center of Central South University's Xiangya Hospital from July to August 2011. All participants provided signed informed consent prior to blood extraction. The Ethics Committees of Xiangya Hospital of Central South University and the Second People's Hospital of Sichuan Province approved the study in accordance with the Declaration of Helsinki.

In a previous study, ${ }^{9}$ seven tSNPs of four genes were found to be significantly associated with the risk of breast cancer when analyzed using a codominant model in unselected cases, including rs12951053 located in TP53, rs1805812, rs2735385 and rs6999227 in NBS1, rs16945628 and rs7220719 in BRIP1, and rs2299941 in PTEN. Finally, in the present study, we analyzed the association between these tSNPs and breast cancer risk using tumor pathological characteristics and body mass index (BMI). SNP genotyping was performed using a custom-by-design 2x48-Plex SNPscan kit (cat\#: G0104, Genesky Biotechnologies Inc., Shanghai, China), as described in a previous study. ${ }^{9,10}$ In total, 728 patients and 671 controls were successfully genotyped for additional analysis.

\section{Clinical Data}

BMI was calculated from patient bodyweight and height, BMI=weight $(\mathrm{kg}) /$ height squared $\left(\mathrm{m}^{2}\right)$. In accordance with the World Health Organization guidelines, ${ }^{11}$ patients were categorized into three groups: under-weight: BMI $<18.5 \mathrm{~kg} / \mathrm{m}^{2}$; normal weight: $18.5 \leq \mathrm{BMI}<25 \mathrm{~kg} / \mathrm{m}^{2}$; or overweight: $\mathrm{BMI} \geqq 25 \mathrm{~kg} / \mathrm{m}^{2}$. Due to a number of missing data points for bodyweight and height, a total of only 496 cases were included in the BMI analysis.

All tissue samples of the patients were analyzed using immunohistochemistry (IHC) by experienced pathologists, including staining of the following markers: ER, PR, Ki67 (cell proliferation marker) and HER2. In accordance with the National Comprehensive Cancer Network (NCCN) Clinical Practice Guidelines for Breast Cancer, Version 3.2020, breast cancer tumors in which at least $1 \%$ of cells stained positive for ER or PR were considered ERor PR-positive. Tumors were classified as HER2-positive if they scored $3+$ using IHC, defined as uniform membrane staining for HER2 in $10 \%$ or more of tumor cells, or demonstrating HER2 gene amplification as observed by in situ hybridization (ISH). In the present study, if the HER2 marker was scored $2+$ by IHC, the sample was further assessed by fluorescence in situ hybridization (FISH). As recommended by the St Gallen International Expert Consensus on the Primary Therapy of Early Breast Cancer 2013, ${ }^{12}$ tumors were grouped into four subtypes 
(Luminal A, Luminal B, HER2 positive and Triple negative) depending on the status of four markers (ER, PR, Ki67 and HER2) (Table 1). A total of 591 cases were included for subtype analysis because patients who scored HER2 $2+$ by IHC without further assessment by FISH were excluded and so some data are missing.

Tumor TNM (T,tumor; N,lymph node; M,metastasis) staging and lymph node status were examined according to the American Joint Committee on Cancer (AJCC) standards in 2010. Due to missing data, a total of 550 cases were investigated for TNM staging analysis and 589 cases for lymph node analysis.

\section{Statistical Methods}

Unconditional logistic regression was used to estimate odds ratios (OR) and associated 95\% confidence intervals (CI) as measures of association between genotypes and risk of breast cancer subtypes, TNM staging, lymph node status and BMI (comparing case subtypes to common homozygote controls). Statistical analysis was conducted using SPSS v.24.0 software.

\section{Results}

\section{Association Between tSNPs and Breast Cancer Risk by Body Mass Index}

As described in Table 2 , in the BMI $\geqq 25 \mathrm{~kg} / \mathrm{m}^{2}$ group, compared with the common homozygote, the heterozygote C/A of tSNP rs12951053 in TP53 displayed an increased risk of breast cancer $(\mathrm{OR}=1.50,95 \% \mathrm{CI}$ : 1.02-2.21, $P=0.041)$. The same trend also was observed in tSNP rs16945628 in BRIP1, but for the uncommon homozygote T/T (OR=1.92, 95\% CI: 1.13-3.26, $P=0.015)$. Conversely, the uncommon homozygote $\mathrm{G} / \mathrm{G}$ of tSNP rs2299941 in PTEN demonstrated a decreased risk of breast cancer
(OR=0.49, 95\% CI: 0.24-0.99, $P=0.042)$. No significant associations were observed in the $\mathrm{BMI}<18.5 \mathrm{~kg} / \mathrm{m}^{2}$ group.

In the $18.5 \leq \mathrm{BMI}<25 \mathrm{~kg} / \mathrm{m}^{2}$ group, five tSNPs displayed a decreased risk of breast cancer compared with the common homozygote, including two heterozygous genotypes (C/T of tSNP rs1805812 in NBS1, OR=0.68, 95\% CI: $0.50-0.94, P=0.019$; and G/A of tSNP rs7220719 in BRIP1, OR=0.67, 95\% CI: $0.49-0.90, P=0.008$ ), the uncommon homozygote $\mathrm{A} / \mathrm{A}$ of tSNP rs2735385 in NBS1 (OR=0.63, 95\% CI: 0.42-0.95, $P=0.026)$, and two tSNPs containing both heterozygous genotypes and uncommon homozygous genotypes $(\mathrm{G} / \mathrm{C}$ and $\mathrm{C} / \mathrm{C}$ of tSNP rs6999227 of NBS1, OR=0.75, 95\% CI: 0.56-0.99, $P=0.049$; OR=0.64, 95\% CI: $0.43-0.95, P=0.027$, respectively, G/A and $\mathrm{G} / \mathrm{G}$ of tSNP rs2299941 of PTEN, $\mathrm{OR}=0.67,95 \%$ CI: $0.51-0.89, P=0.005 ; \mathrm{OR}=0.60,95 \%$ CI: $0.38-0.94, P=0.025$, respectively) (Table 2 ).

\section{Association Between tSNPs and Breast Cancer Risk by Tumor Pathological Characteristics}

As presented in Table 3, luminal A (44.3\%) was the subtype of breast cancer in the highest proportion in the present study, followed by triple-negative $(27.8 \%)$, the lowest being the HER-2 positive/HR negative subtype $(5.8 \%)$, similar to the proportion in our previous reports. ${ }^{13}$

Four tSNPs were negatively associated with luminal $\mathrm{B}$ breast cancer compared with the common homozygote (Table 3). Of these, two tSNPs in NBS1 (tSNP rs2735385 and rs6999227) contained both heterozygous genotypes and uncommon homozygous genotypes that displayed a negative association, $\mathrm{OR}=0.63,95 \% \mathrm{CI}$ : $0.42-0.94$, $P=0.024$ and $\mathrm{OR}=0.45,95 \% \mathrm{CI}: 0.24-0.83, P=0.010$ for the $\mathrm{C} / \mathrm{A}$ and $\mathrm{A} / \mathrm{A}$ genotypes of $\mathrm{rs} 2735385$, respectively;

Table I Surrogate Definitions of Intrinsic Subtypes of Breast Cancer

\begin{tabular}{|l|l|}
\hline Intrinsic Subtype & Clinico-Pathologic Surrogate Definition \\
\hline Luminal A & All of :ER and PR positive (PR $\geq 20 \%$ positive), HER2 negative, low Ki-67 (<I4\%) \\
\hline Luminal B & $\begin{array}{c}\text { Luminal B (HER2 negative): } \\
\text { ER positive, HER2 negative and at least one of: high Ki-67( } \geq 14 \%), \text { PR negative or low (<20\% positive) } \\
\text { Luminal B (HER2 positive): } \\
\text { ER positive, HER2 over-expressed or amplified, any Ki-67, and any PR }\end{array}$ \\
\hline HER2 positive & HER2 over-expressed or amplified, ER and PR absent \\
\hline Triple negative & ER and PR absent, HER2 negative \\
\hline
\end{tabular}

Note: Data from Goldhirsch et al. ${ }^{12}$ 
Table 2 Odds Ratios and 95\% Confidence Intervals for Breast Cancer Risk by Body Mass Index

\begin{tabular}{|c|c|c|c|c|c|c|c|c|c|c|c|c|}
\hline \multirow[t]{3}{*}{ Gene } & \multirow[t]{3}{*}{ tSNP } & \multirow[t]{3}{*}{ Genotype } & \multirow{3}{*}{$\begin{array}{l}\text { Controls } \\
\mathbf{N}\end{array}$} & \multicolumn{9}{|c|}{$\operatorname{BMI}\left(\mathrm{kg} / \mathrm{m}^{2}\right)$} \\
\hline & & & & \multicolumn{3}{|c|}{$<18.5$} & \multicolumn{3}{|c|}{ 18.5-24.99 } & \multicolumn{3}{|l|}{$\geq 25$} \\
\hline & & & & $\mathbf{N}$ & $\mathrm{OR}^{\mathrm{a}}(95 \% \mathrm{Cl})$ & $P$-value ${ }^{b}$ & $\mathbf{N}$ & $\mathrm{OR}^{\mathrm{a}}(95 \% \mathrm{Cl})$ & $P$-value ${ }^{b}$ & $\mathbf{N}$ & $\mathrm{OR}^{\mathrm{a}}(95 \% \mathrm{Cl})$ & $P$-value ${ }^{b}$ \\
\hline \multirow[t]{3}{*}{ TP53 } & rs 12951053 & $\mathrm{~A} / \mathrm{A}$ & 331 & 12 & 1.00 & & 153 & 1.00 & & 55 & 1.00 & \\
\hline & & $\mathrm{C} / \mathrm{A}$ & 273 & 11 & $1.11(0.48-2.56)$ & 0.804 & 156 & $1.24(0.94-1.63)$ & 0.130 & 68 & $1.50(1.02-2.21)$ & 0.041 \\
\hline & & $\mathrm{C} / \mathrm{C}$ & 67 & 2 & $0.82(0.18-3.76)$ & 0.802 & 23 & $0.74(0.45-1.24)$ & 0.253 & 15 & $1.35(0.72-2.53)$ & 0.351 \\
\hline \multirow[t]{9}{*}{ NBSI } & rs 1805812 & $T / T$ & 470 & 20 & 1.00 & & 254 & 1.00 & & 100 & 1.00 & \\
\hline & & $\mathrm{C} / \mathrm{T}$ & 184 & 5 & $0.64(0.24-1.73)$ & 0.373 & 68 & $0.68(0.50-0.94)$ & 0.019 & 34 & $0.87(0.57-1.33)$ & 0.515 \\
\hline & & $\mathrm{C} / \mathrm{C}$ & 16 & 0 & 1 & 1 & 11 & I.27(0.58-2.78) & 0.546 & 4 & $1.18(0.39-3.59)$ & 0.777 \\
\hline & rs2735385 & $\mathrm{C} / \mathrm{C}$ & 210 & 8 & 1.00 & & 127 & 1.00 & & 54 & 1.00 & \\
\hline & & $\mathrm{C} / \mathrm{A}$ & 345 & 14 & $1.07(0.44-2.58)$ & 0.889 & 161 & $0.77(0.58-1.03)$ & 0.078 & 61 & $0.69(0.46-1.03)$ & 0.069 \\
\hline & & $\mathrm{A} / \mathrm{A}$ & 116 & 3 & $0.68(0.18-2.61)$ & 0.571 & 44 & $0.63(0.42-0.95)$ & 0.026 & 23 & $0.77(0.45-1.32)$ & 0.343 \\
\hline & rs6999227 & $\mathrm{G} / \mathrm{G}$ & 200 & 7 & 1.00 & & 124 & 1.00 & & 49 & 1.00 & \\
\hline & & $\mathrm{G} / \mathrm{C}$ & 344 & 14 & $1.16(0.46-2.93)$ & 0.749 & 159 & $0.75(0.56-0.99)$ & 0.049 & 64 & $0.76(0.50-1.15)$ & 0.188 \\
\hline & & $\mathrm{C} / \mathrm{C}$ & 126 & 4 & $0.91(0.26-3.16)$ & 0.878 & 50 & $0.64(0.43-0.95)$ & 0.027 & 25 & $0.81(0.48-1.38)$ & 0.436 \\
\hline \multirow[t]{3}{*}{ BRIPI } & rs 16945628 & $\mathrm{C} / \mathrm{C}$ & 271 & 10 & 1.00 & & 154 & 1.00 & & 46 & 1.00 & \\
\hline & & $\mathrm{C} / \mathrm{T}$ & 313 & 12 & $1.04(0.44-2.44)$ & 0.930 & 135 & $0.76(0.57-1.01)$ & 0.056 & 64 & $1.21(0.80-1.82)$ & 0.376 \\
\hline & & $T / T$ & 86 & 3 & $0.95(0.25-3.51)$ & 0.933 & 43 & $0.88(0.58-1.33)$ & 0.546 & 28 & $1.92(1.13-3.26)$ & 0.015 \\
\hline \multirow{6}{*}{ PTEN } & rs7220719 & $\mathrm{G} / \mathrm{G}$ & 429 & 17 & 1.00 & & 235 & 1.00 & & 88 & 1.00 & \\
\hline & & $\mathrm{G} / \mathrm{A}$ & 217 & 6 & $0.70(0.27-1.80)$ & 0.453 & 79 & $0.67(0.49-0.90)$ & 0.008 & 41 & $0.92(0.61-1.38)$ & 0.691 \\
\hline & & $\mathrm{A} / \mathrm{A}$ & 25 & 2 & $2.02(0.44-9.23)$ & 0.355 & 18 & $1.31(0.70-2.50)$ & 0.391 & 9 & $1.76(0.79-3.89)$ & 0.161 \\
\hline & rs229994l & $\mathrm{A} / \mathrm{A}$ & 268 & 12 & 1.00 & & 168 & 1.00 & & 65 & 1.00 & \\
\hline & & $G / A$ & 314 & 11 & $0.78(0.34-1.80)$ & 0.563 & 132 & $0.67(0.51-0.89)$ & 0.005 & 63 & $0.83(0.56-1.21)$ & 0.331 \\
\hline & & G/G & 85 & 2 & $0.53(0.12-2.40)$ & 0.398 & 32 & $0.60(0.38-0.94)$ & 0.025 & 10 & $0.49(0.24-0.99)$ & 0.042 \\
\hline
\end{tabular}

Notes: ${ }^{\mathrm{a} C o m p a r e d}$ with common homozygote by logistic regression analysis. ${ }^{\mathrm{P}} \mathrm{P}$ value for every genetype when compared with common homozygote by logistic regression analysis. Bold formatted text values indicate statistically significant results $(P<0.05)$.

Abbreviations: N, number; OR, odds ratio; BMI, body mass index; tSNP, tagging single nucleotide polymorphism.

$\mathrm{OR}=0.58,95 \% \mathrm{CI}: 0.39-0.88, P=0.009$ and $\mathrm{OR}=0.51$, 95\% CI: $0.29-0.91, P=0.020$ for the $\mathrm{G} / \mathrm{C}$ and $\mathrm{C} / \mathrm{C}$ genotypes of rs6999227, respectively (Table 3 ). The tSNP rs1805812 in NBS1 contained only a heterozygous $\mathrm{C} / \mathrm{T}$ genotype that exhibited a negative association $(\mathrm{OR}=0.51$, 95\% CI: $0.31-0.83, P=0.007)$. The tSNP rs2299941 in PTEN contained only the uncommon homozygous genotype $\mathrm{G} / \mathrm{G}$ that displayed a negative association $(\mathrm{OR}=0.41$, 95\% CI: $0.19-0.90, P=0.022)$. Each single allele of the four tSNPs also exhibited a negative association $(\mathrm{OR}=0.60-0.73)$ (Table 3).

Several tSNPs were strongly associated with triplenegative breast cancer (Table 3). Patients with the $\mathrm{C} / \mathrm{A}$ heterozygote tSNP rs12951053 located in TP53 exhibited a risk of triple-negative breast cancer 1.50-fold greater (95\% CI: $1.05-2.15, P=0.026$ ) than those with the common homozygote A/A. The uncommon homozygous genotype A/A of tSNP rs7220719 in BRIP1 displayed a higher risk than that described above $(\mathrm{OR}=2.13,95 \%$ CI: 1.05-4.29, $P=0.032$ ). The tSNPs, rs1805812 and rs2735385 of NBS1 displayed a negative association with triple-negative breast cancer compared with the common homozygote $(\mathrm{OR}=0.61,95 \% \mathrm{CI}: 0.40-0.93, P=0.022$ for genotype $\mathrm{C} / \mathrm{T}$ of $\mathrm{rs} 1805812$; $\mathrm{OR}=0.64,95 \% \mathrm{CI}$ : $0.45-$ 0.93, $P=0.019$ for genotype $\mathrm{C} / \mathrm{A}$ and $\mathrm{OR}=0.49,95 \% \mathrm{CI}$ : $0.28-0.86, P=0.011$ for genotype A/A of rs2735385). Each single allele of the two tSNPs also exhibited a negative association $(\mathrm{OR}=0.66,95 \% \quad \mathrm{CI}: \quad 0.46-0.96, \quad P=0.029$; $\mathrm{OR}=0.69$, 95\% CI: $0.54-0.89, P=0.004$, respectively). Only one allele of two other tSNPs showed a negative association with triple-negative breast cancer $(\mathrm{OR}=0.77$, 95\% CI: $0.60-0.99, P=0.037$ for rs6999227 in NBS1 and $\mathrm{OR}=0.74$, 95\% CI: $0.57-0.97, P=0.027$ for rs 2299941 in PTEN) (Table 3).

Only two tSNPs with a heterozygous genotype exhibited a negative association with luminal A breast cancer, $\mathrm{OR}=0.70,95 \% \mathrm{CI}: 0.50-0.97, P=0.030$ for genotype $\mathrm{G} / \mathrm{A}$ of rs7220719 in BRIP1 and OR=0.73, 95\% CI: 0.54-0.98, $P=0.039$ for genotype $\mathrm{G} / \mathrm{A}$ of rs2299941 in PTEN (Table 3). The same trend was observed for every single allele of 


\begin{tabular}{|c|c|c|c|c|c|c|c|c|c|c|}
\hline \multirow{3}{*}{ 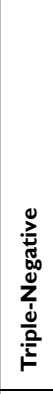 } & \multirow{3}{*}{$\begin{array}{l}\frac{\pi}{0} \\
\frac{\pi}{11} \\
z\end{array}$} & $\stackrel{a}{a}$ & 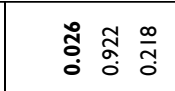 & 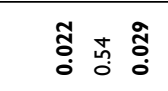 & $\begin{array}{l}\frac{0}{0} \overline{0} \\
0 \\
0\end{array}$ & 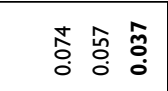 & 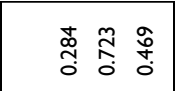 & 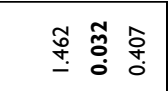 & 哭 荇 & 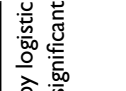 \\
\hline & & 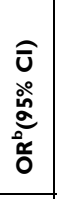 & 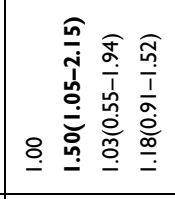 & 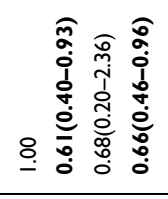 & 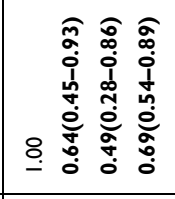 & 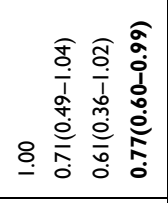 & 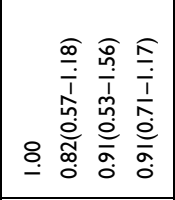 & 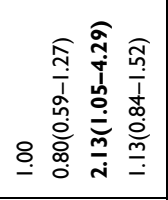 & 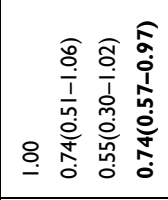 & 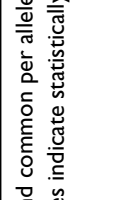 \\
\hline & & $\mathbf{z}$ & 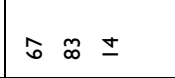 & $\stackrel{\stackrel{9}{m} \bar{m} m}{m}$ & R & סָ & ल $\hat{\sigma} \bar{N}$ & 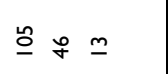 & $\infty \quad$ o $\quad \pm$ & 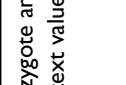 \\
\hline \multirow{3}{*}{ 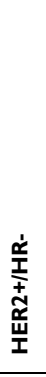 } & \multirow[b]{3}{*}{ 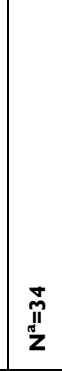 } & : & 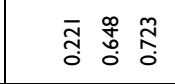 & 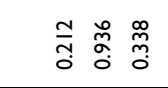 & 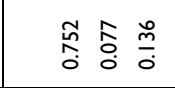 & 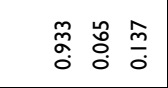 & 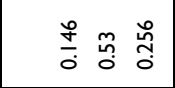 & $\begin{array}{cc}\stackrel{\infty}{\infty} & \stackrel{\leftrightarrow}{0} \\
0 & \stackrel{0}{0}\end{array}$ & 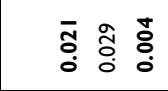 & ¿. \\
\hline & & 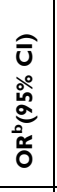 & 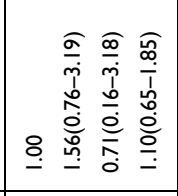 & 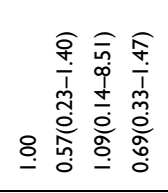 & 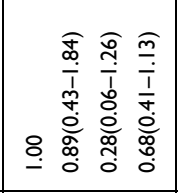 & 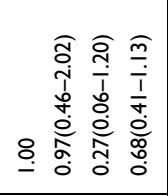 & 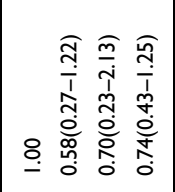 & 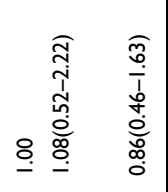 & 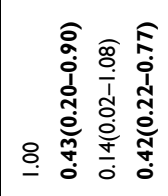 & 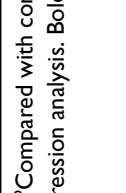 \\
\hline & & $\mathbf{z}$ & $\pm \stackrel{\infty}{\sim}$ & $\hat{\wedge} \circ-$ & $\underline{m} \sim$ & $\simeq \stackrel{N}{ }$ & $\underline{\infty} \simeq$ & $\approx \simeq 0$ & $\approx=-$ & \\
\hline \multirow{3}{*}{ 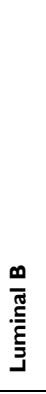 } & \multirow{3}{*}{$\begin{array}{l}\text { o } \\
\frac{m}{11} \\
\text { z }\end{array}$} & : & 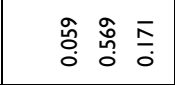 & 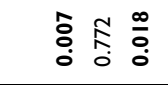 & 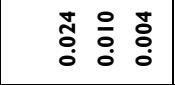 & 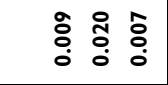 & 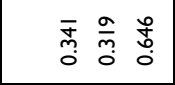 & 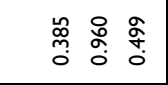 & 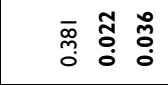 & $\subseteq$ \\
\hline & & 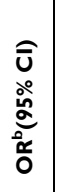 & 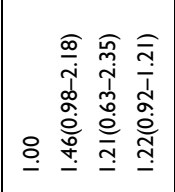 & 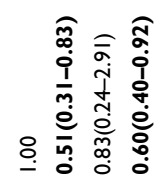 & 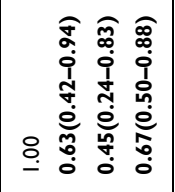 & 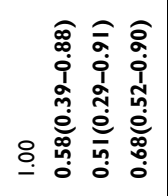 & 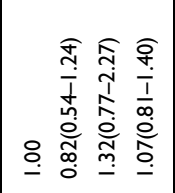 & 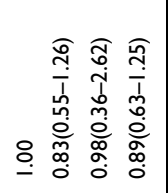 & 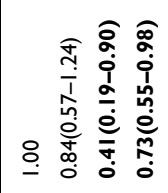 & 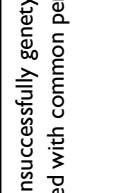 \\
\hline & & $\mathbf{z}$ & 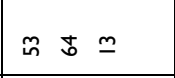 & $\stackrel{\circ}{\circ} \bar{s} m$ & in on \pm & i̊ i⿱ & 负 $\approx$ in & $\infty$ 角 & $\overline{6}: \stackrel{\infty}{\circ}$ & \\
\hline \multirow{3}{*}{ 选 } & \multirow[b]{3}{*}{$\begin{array}{l}\bar{i} \\
\text { in } \\
\mathbf{z}\end{array}$} & $\stackrel{a}{a}$ & 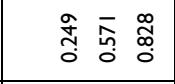 & 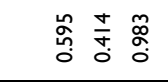 & $\frac{f}{0} \frac{m}{0} \frac{\bar{m}}{0}$ & 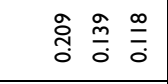 & 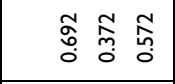 & 总 导 & 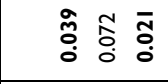 & 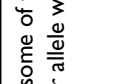 \\
\hline & & 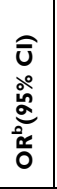 & 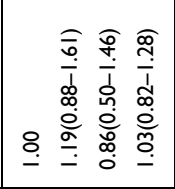 & 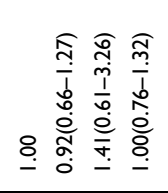 & 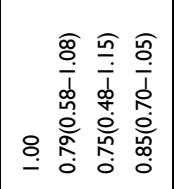 & 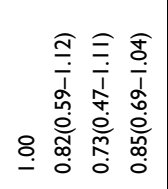 & 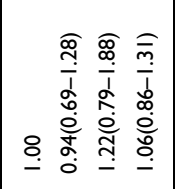 & 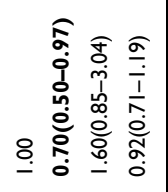 & 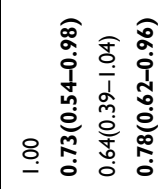 & 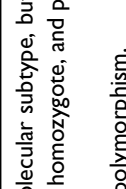 \\
\hline & & $\mathbf{z}$ & $\overline{\underline{I}} \stackrel{0}{=} \overline{\mathrm{u}}$ & $\stackrel{\underline{\omega}}{\circ} \sigma$ & $\approx \stackrel{2}{\simeq} q$ & 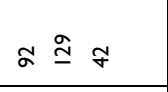 & $\stackrel{\circ}{\varrho} \stackrel{ }{=} \sigma$ & $\underline{\simeq} \pm \simeq$ & $\stackrel{\infty}{\simeq} \stackrel{\circ}{9}$ & $\stackrel{\Xi}{c}$ \\
\hline 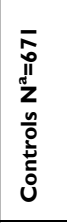 & & $\mathbf{z}$ & 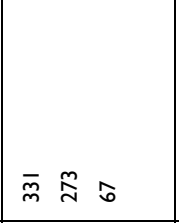 & 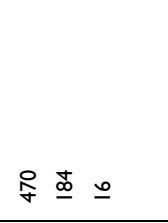 & $\stackrel{\circ}{\sim} \stackrel{\text { 号 }}{=}$ & 品 声 & $\bar{\lambda} \frac{m}{m} \infty$ & 守 & 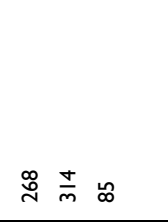 & 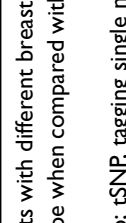 \\
\hline 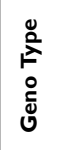 & & & 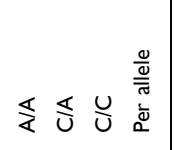 & 占与 & 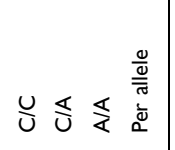 & 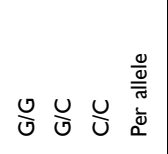 & U与 & 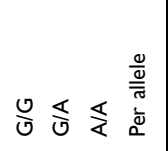 & 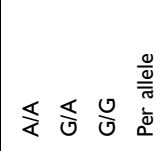 & 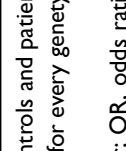 \\
\hline $\begin{array}{l}\sum_{y}^{2} \\
y\end{array}$ & & & $\begin{array}{l}\frac{n}{\hat{o}} \\
\frac{\hat{\alpha}}{\mathfrak{n}} \\
\frac{\mathfrak{n}}{2}\end{array}$ & 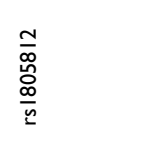 & 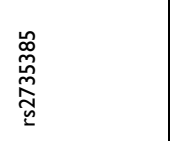 & 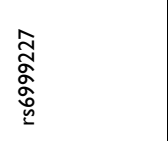 & 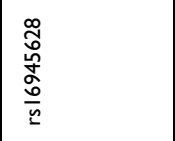 & 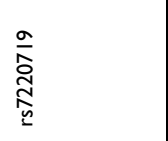 & 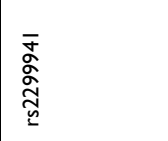 & 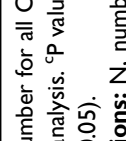 \\
\hline 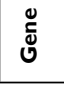 & & & 啇 & 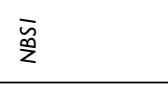 & & & 高 & & 总 & z \\
\hline
\end{tabular}


rs2299941 (OR=0.78, 95\% CI: 0.62-0.96, $P=0.021)$. The heterozygote G/A of rs2299941 in PTEN also exhibited a negative association with HER-2 positive/HR negative breast cancer $(\mathrm{OR}=0.43,95 \% \mathrm{CI}: 0.20-0.90, P=0.021)$, every single allele displaying the same trend $(\mathrm{OR}=0.42$, 95\% CI: 0.22-0.77, $P=0.004$ ) (Table 3).

As displayed in Table 4, three tSNPs (rs2735385 and rs6999227 in NBS1, rs2299941 in PTEN) in both heterozygous genotypes and the uncommon homozygous genotypes, displayed a negative association with stage II breast cancer compared with the common homozygote $(\mathrm{OR}=0.71,95 \%$ CI: $0.53-0.95, P=0.021$ and $\mathrm{OR}=0.58$, 95\% CI: $0.38-0.89, P=0.011$ for genotypes $\mathrm{C} / \mathrm{A}$ and $\mathrm{A} / \mathrm{A}$ of rs2735385, respectively; OR $=0.70,95 \%$ CI: $0.52-0.94$, $P=0.018$ and $\mathrm{OR}=0.61,95 \%$ CI: $0.41-0.92, P=0.016$ for genotypes $\mathrm{G} / \mathrm{C}$ and $\mathrm{C} / \mathrm{C}$ of $\mathrm{rs} 6999227$, respectively; $\mathrm{OR}=0.73,95 \%$ CI: $0.55-0.97, P=0.028$ and $\mathrm{OR}=0.48$, 95\% CI: $0.29-0.79, P=0.003$ for genotypes $\mathrm{G} / \mathrm{A}$ and $\mathrm{G} / \mathrm{G}$ of rs2299941, respectively). The two other tSNPs with only heterozygous genotypes also displayed a negative association with stage II breast cancer ( $\mathrm{OR}=0.70,95 \%$ CI: $0.50-0.96, P=0.027$ for genotype $\mathrm{C} / \mathrm{T}$ of $\mathrm{rs} 1805812$ in NBS1 and OR $=0.68,95 \%$ CI: $0.50-0.93, P=0.015$ for genotype G/A of rs7220719 in BRIP1). Only one heterozygote, the genotype C/A of tSNP rs12951053 located in TP53 exhibited a positive association with stage II breast cancer $(\mathrm{OR}=1.54,95 \% \mathrm{CI}: 1.16-2.04, P=0.003)$.

In stage III breast cancer, only three tSNPs were found to have a negative association, a heterozygous genotype and an uncommon homozygote genotype $(\mathrm{OR}=0.61,95 \%$ CI: $0.42-0.88, P=0.007$ and $\mathrm{OR}=0.53,95 \%$ CI: $0.31-0.90$, $P=0.017$ for genotypes $\mathrm{C} / \mathrm{A}$ and $\mathrm{A} / \mathrm{A}$ of rs 2735385 in NBS1, respectively), the other a heterozygous genotype ( $\mathrm{OR}=0.62,95 \%$ CI: $0.43-0.88, P=0.008$ for genotype $\mathrm{G} / \mathrm{A}$ of rs2299941 in PTEN) and another, an uncommon homozygote ( $\mathrm{OR}=0.47,95 \% \mathrm{CI}: 0.27-0.81, P=0.006$ for genotype $\mathrm{C} / \mathrm{C}$ of rs6999227 in NBS1) (Table 4). No significant associations with stage I breast cancer were identified.

Analysis of the association between tSNPs and lymph node status of breast cancer indicated that a number of

Table 4 Odds Ratios and 95\% Confidence Intervals for the Association Between tSNPs and Each TNM Staging

\begin{tabular}{|c|c|c|c|c|c|c|c|c|c|c|c|c|}
\hline \multirow[t]{3}{*}{ Gene } & \multirow[t]{3}{*}{ tSNP } & \multirow[t]{3}{*}{ Genotype } & \multirow{3}{*}{$\begin{array}{l}\text { Controls } \\
\mathbf{N}\end{array}$} & \multicolumn{9}{|c|}{ TNM Staging } \\
\hline & & & & \multicolumn{3}{|c|}{ Stage I } & \multicolumn{3}{|c|}{ Stage II } & \multicolumn{3}{|c|}{ Stage III } \\
\hline & & & & $\mathbf{N}$ & $\mathrm{OR}^{\mathrm{a}}(95 \% \mathrm{Cl})$ & $P$-value ${ }^{b}$ & $\mathbf{N}$ & $\mathrm{OR}^{\mathrm{a}}(95 \% \mathrm{Cl})$ & $P$-value ${ }^{b}$ & $\mathbf{N}$ & $\mathrm{OR}^{\mathrm{a}}(95 \% \mathrm{Cl})$ & $P$-value ${ }^{b}$ \\
\hline \multirow[t]{3}{*}{ TP53 } & rs 12951053 & $\mathrm{~A} / \mathrm{A}$ & 331 & 28 & 1.00 & & 126 & 1.00 & & 83 & 1.00 & \\
\hline & & $\mathrm{C} / \mathrm{A}$ & 273 & 30 & $1.30(0.76-2.23)$ & 0.341 & 160 & $1.54(1.16-2.04)$ & 0.003 & 76 & $1.11(0.78-1.58)$ & 0.558 \\
\hline & & $\mathrm{C} / \mathrm{C}$ & 67 & 3 & $0.53(0.16-1.79)$ & 0.299 & 28 & $1.10(0.68-1.79)$ & 0.707 & 14 & $0.83(0.45-1.56)$ & 0.567 \\
\hline \multirow[t]{9}{*}{ NBSI } & rs 1805812 & $T / T$ & 470 & 46 & 1.00 & & 239 & 1.00 & & 133 & 1.00 & \\
\hline & & $\mathrm{C} / \mathrm{T}$ & 184 & 14 & $0.78(0.42-1.45)$ & 0.427 & 65 & $0.70(0.50-0.96)$ & 0.027 & 38 & $0.73(0.49-1.09)$ & 0.121 \\
\hline & & $\mathrm{C} / \mathrm{C}$ & 16 & 1 & $0.64(0.08-4.93)$ & 0.664 & 12 & $1.48(0.69-3.17)$ & 0.316 & 2 & $0.44(0.10-1.95)$ & 0.267 \\
\hline & rs2735385 & $\mathrm{C} / \mathrm{C}$ & 210 & 26 & 1.00 & & 127 & 1.00 & & 75 & 1.00 & \\
\hline & & $\mathrm{C} / \mathrm{A}$ & 345 & 27 & $0.63(0.36-1.11)$ & 0.109 & 148 & $0.71(0.53-0.95)$ & 0.021 & 75 & $0.61(0.42-0.88)$ & 0.007 \\
\hline & & $\mathrm{A} / \mathrm{A}$ & 116 & 8 & $0.56(0.24-1.27)$ & 0.159 & 41 & $0.58(0.38-0.89)$ & 0.011 & 22 & $0.53(0.31-0.90)$ & 0.017 \\
\hline & rs6999227 & G/G & 200 & 24 & 1.00 & & 122 & 1.00 & & 68 & 1.00 & \\
\hline & & $\mathrm{G} / \mathrm{C}$ & 344 & 28 & $0.68(0.38-1.20)$ & 0.182 & 147 & $0.70(0.52-0.94)$ & 0.018 & 85 & $0.73(0.51-1.05)$ & 0.085 \\
\hline & & $\mathrm{C} / \mathrm{C}$ & 126 & 9 & $0.60(0.27-1.32)$ & 0.199 & 47 & $0.61(0.41-0.92)$ & 0.016 & 20 & $0.47(0.27-0.81)$ & 0.006 \\
\hline \multirow[t]{6}{*}{ BRIPI } & rs 16945628 & $\mathrm{C} / \mathrm{C}$ & 271 & 26 & 1.00 & & 139 & 1.00 & & 65 & 1.00 & \\
\hline & & $\mathrm{C} / \mathrm{T}$ & 313 & 29 & $0.97(0.56-1.68)$ & 0.902 & 132 & $0.82(0.62-1.10)$ & 0.183 & 75 & $1.00(0.69-1.45)$ & 0.996 \\
\hline & & $T / T$ & 86 & 6 & $0.73(0.29-1.83)$ & 0.496 & 43 & $0.98(0.64-1.48)$ & 0.905 & 33 & $1.60(0.99-2.60)$ & 0.056 \\
\hline & rs7220719 & G/G & 429 & 38 & 1.00 & & 220 & 1.00 & & 104 & 1.00 & \\
\hline & & $\mathrm{G} / \mathrm{A}$ & 217 & 18 & $0.94(0.52-1.68)$ & 0.826 & 76 & $0.68(0.50-0.93)$ & 0.015 & 59 & $1.12(0.78-1.61)$ & 0.531 \\
\hline & & $\mathrm{A} / \mathrm{A}$ & 25 & 5 & $2.26(0.82-6.24)$ & 0.107 & 19 & $1.48(0.80-2.75)$ & 0.21 & 10 & $1.65(0.77-3.54)$ & 0.195 \\
\hline \multirow[t]{3}{*}{ PTEN } & rs229994l & $\mathrm{A} / \mathrm{A}$ & 268 & 23 & 1.00 & & 157 & 1.00 & & 90 & 1.00 & \\
\hline & & $\mathrm{G} / \mathrm{A}$ & 314 & 33 & $1.23(0.70-2.14)$ & 0.475 & 134 & $0.73(0.55-0.97)$ & 0.028 & 65 & $0.62(0.43-0.88)$ & 0.008 \\
\hline & & G/G & 85 & 5 & $0.69(0.25-1.86)$ & 0.456 & 24 & $0.48(0.29-0.79)$ & 0.003 & 18 & $0.63(0.36-1.11)$ & 0.106 \\
\hline
\end{tabular}

Notes: ${ }^{a}$ Compared with common homozygote by logistic regression analysis. ${ }^{\mathrm{b}} \mathrm{P}$ value for every genetype when compared with common homozygote by logistic regression analysis. Bold formatted text values indicate statistically significant results $(P<0.05)$.

Abbreviations: N, number; OR, odds ratio; TNM, tumor lymph node metastasis; tSNP, tagging single nucleotide polymorphism. 
tSNPs displayed a negative association with lymph nodenegative breast cancer (rs1805812, rs2735385 and rs6999227 in NBS1, rs7220719 in BRIP1 and rs2299941 in PTEN, OR=0.51-0.73) (Table 5). Only one heterozygous genotype, C/A of tSNP rs12951053 in TP53 exhibited a positive association with lymph node-negative breast cancer $(\mathrm{OR}=1.43,95 \% \mathrm{CI}$ : $1.08-1.91, P=0.013)$. We also found three tSNPs (rs2735385 and rs6999227 in NBS1 and rs2299941 in PTEN, OR=0.53-0.67) that were negatively associated with breast cancers having $1-3$ positive nodes (Table 5). The same trend was observed in breast cancer with more than three positive nodes (rs1805812 and rs2735385 in NBS1 and rs2299941 in PTEN, OR=0.53-0.61) (Table 5).

\section{Discussion}

With the development of breast cancer susceptibility gene panels, previous studies have demonstrated that genetic factors can also influence tumor subtype, finding that in
TNBC metastatic patients with a germline BRCA mutation, monotherapy with the PARP inhibitor olaparib provided a significant benefit over standard therapy. ${ }^{3,4}$ Thus, understanding the associations between genetic polymorphisms and clinicopathological features of breast cancer may ultimately result in improvements in prevention, early detection, and treatment. In a previous study, we found that seven tagging SNPs (tSNPs) of four genes were significantly associated with breast cancer risk using a codominant model in unselected cases. ${ }^{9}$ In the present study, we evaluated the associations between these tSNPs and breast cancer risk defined by tumor pathological characteristics and BMI.

Compared with the common homozygote, we found that the heterozygote C/A of tSNP rs12951053 located in TP53 and the uncommon homozygote T/T of tSNP rs16945628 located in BRIP1 displayed an increased risk of breast cancer in the BMI $\geqq 25 \mathrm{~kg} / \mathrm{m}^{2}$ group. Conversely, in the $18.5 \leq \mathrm{BMI}<25 \mathrm{~kg} / \mathrm{m}^{2}$ group, five

Table 5 Oddi Ratios and 95\% Confidence Intervals for the Association Between tSNPs and Lymph Node Status

\begin{tabular}{|c|c|c|c|c|c|c|c|c|c|c|c|c|}
\hline \multirow[t]{3}{*}{ Gene } & \multirow[t]{3}{*}{ tSNP } & \multirow[t]{3}{*}{ Genotype } & \multirow{3}{*}{$\begin{array}{l}\text { Controls } \\
\mathbf{N}\end{array}$} & \multicolumn{9}{|c|}{ Number of Positive Nodes } \\
\hline & & & & \multicolumn{3}{|l|}{0} & \multicolumn{3}{|l|}{$1-3$} & \multicolumn{3}{|l|}{$>3$} \\
\hline & & & & $\mathbf{N}$ & $O R^{a}(95 \% \mathrm{CI})$ & $P_{\text {-value }}{ }^{b}$ & $\mathbf{N}$ & $\mathrm{OR}^{\mathrm{a}}(95 \% \mathrm{Cl})$ & $P$-value ${ }^{b}$ & $\mathbf{N}$ & $O^{a}(95 \% \mathrm{CI})$ & $P$-value ${ }^{b}$ \\
\hline \multirow[t]{3}{*}{ TP53 } & \multirow[t]{3}{*}{ rs 12951053} & $\mathrm{~A} / \mathrm{A}$ & 331 & 126 & 1.00 & & 75 & 1.00 & & 54 & 1.00 & \\
\hline & & $\mathrm{C} / \mathrm{A}$ & 273 & 149 & $1.43(1.08-1.91)$ & 0.013 & 77 & $1.25(0.87-1.78)$ & 0.228 & 56 & $1.26(0.84-1.89)$ & 0.269 \\
\hline & & $\mathrm{C} / \mathrm{C}$ & 67 & 28 & $1.10(0.68-1.79)$ & 0.707 & 13 & $0.86(0.45-1.63)$ & 0.637 & 9 & $0.82(0.39-1.75)$ & 0.612 \\
\hline \multirow[t]{9}{*}{ NBSI } & \multirow[t]{3}{*}{ rs 1805812} & $\mathrm{~T} / \mathrm{T}$ & 470 & 236 & 1.00 & & 119 & 1.00 & & 93 & 1.00 & \\
\hline & & $\mathrm{C} / \mathrm{T}$ & 184 & 58 & $0.63(0.45-0.88)$ & 0.006 & 45 & $0.97(0.66-1.42)$ & 0.859 & 21 & $0.58(0.35-0.95)$ & 0.030 \\
\hline & & $\mathrm{C} / \mathrm{C}$ & 16 & 10 & $1.25(0.56-2.79)$ & 0.594 & 2 & $0.49(0.11-2.18)$ & 0.342 & 5 & $1.58(0.57-4.42)$ & 0.380 \\
\hline & \multirow[t]{3}{*}{ rs2735385 } & $\mathrm{C} / \mathrm{C}$ & 210 & 121 & 1.00 & & 69 & 1.00 & & 51 & 1.00 & \\
\hline & & $\mathrm{C} / \mathrm{A}$ & 345 & 140 & $0.70(0.52-0.95)$ & 0.021 & 76 & $0.67(0.46-0.97)$ & 0.033 & 51 & $0.61(0.40-0.93)$ & 0.021 \\
\hline & & $\mathrm{A} / \mathrm{A}$ & 116 & 43 & $0.64(0.43-0.98)$ & 0.037 & 21 & $0.55(0.32-0.94)$ & 0.029 & 16 & $0.57(0.31-1.04)$ & 0.065 \\
\hline & \multirow[t]{3}{*}{ rs6999227 } & $\mathrm{G} / \mathrm{G}$ & 200 & 115 & 1.00 & & 64 & 1.00 & & 45 & 1.00 & \\
\hline & & $\mathrm{G} / \mathrm{C}$ & 344 & 138 & $0.70(0.52-0.94)$ & 0.020 & 83 & $0.75(0.52-1.09)$ & 0.134 & 57 & $0.74(0.48-1.13)$ & 0.160 \\
\hline & & $\mathrm{C} / \mathrm{C}$ & 126 & 51 & $0.70(0.47-1.05)$ & 0.083 & 19 & $0.47(0.27-0.82)$ & 0.007 & 17 & $0.60(0.33-1.09)$ & 0.093 \\
\hline \multirow[t]{6}{*}{$B R I P I$} & \multirow[t]{3}{*}{ rs16945628 } & $\mathrm{C} / \mathrm{C}$ & 271 & 138 & 1.00 & & 64 & 1.00 & & 45 & 1.00 & \\
\hline & & $\mathrm{C} / \mathrm{T}$ & 313 & 123 & $0.77(0.58-1.03)$ & 0.082 & 76 & $1.03(0.7 I-1.49)$ & 0.883 & 53 & $1.02(0.66-1.57)$ & 0.929 \\
\hline & & $\mathrm{T} / \mathrm{T}$ & 86 & 41 & $0.94(0.61-1.43)$ & 0.761 & 26 & $1.28(0.76-2.15)$ & 0.348 & 21 & $\mathrm{I} .47(0.83-2.6 \mathrm{I})$ & 0.185 \\
\hline & \multirow[t]{3}{*}{ rs7220719 } & $\mathrm{G} / \mathrm{G}$ & 429 & 207 & 1.00 & & 107 & 1.00 & & 78 & 1.00 & \\
\hline & & $\mathrm{G} / \mathrm{A}$ & 217 & 76 & $0.73(0.53-0.99)$ & 0.042 & 52 & $0.96(0.66-1.39)$ & 0.832 & 33 & $0.84(0.54-1.30)$ & 0.424 \\
\hline & & $\mathrm{A} / \mathrm{A}$ & 25 & 20 & $1.66(0.90-3.05)$ & 0.102 & 7 & $1.12(0.47-2.67)$ & 0.793 & 8 & $1.76(0.77-4.04)$ & 0.178 \\
\hline \multirow[t]{3}{*}{ PTEN } & \multirow[t]{3}{*}{ rs229994I } & $\mathrm{A} / \mathrm{A}$ & 268 & 136 & 1.00 & & 90 & 1.00 & & 66 & 1.00 & \\
\hline & & $G / A$ & 314 & 145 & $0.91(0.68-1.21)$ & 0.517 & 61 & $0.58(0.40-0.83)$ & 0.003 & 41 & $0.53(0.35-0.81)$ & 0.003 \\
\hline & & $\mathrm{G} / \mathrm{G}$ & 85 & 22 & $0.51(0.31-0.85)$ & 0.009 & 15 & $0.53(0.29-0.96)$ & 0.033 & 12 & $0.57(0.30-1.11)$ & 0.096 \\
\hline
\end{tabular}

Notes: ${ }^{\mathrm{a} C}$ Compared with common homozygote by logistic regression analysis. ${ }^{\mathrm{b}} \mathrm{P}$ value for every genetype when compared with common homozygote by logistic regression analysis. Bold formatted text values indicate statistically significant results $(P<0.05)$.

Abbreviations: $\mathrm{N}$, number; OR, odds ratio; tSNP, tagging single nucleotide polymorphism. 
tSNPs exhibited a decreased risk of breast cancer compared with the common homozygote, including heterozygous genotypes and uncommon homozygotes $(\mathrm{C} / \mathrm{T}$ of tSNP rs1805812, A/A of tSNP rs2735385, G/C and $\mathrm{C} / \mathrm{C}$ of tSNP rs6999227 located in NBS1 and G/A of tSNP rs7220719 located in BRIP1). In addition, the uncommon homozygote $\mathrm{G} / \mathrm{G}$ of tSNP rs2299941 located in PTEN displayed a decreased risk of breast cancer both in the $18.5 \leq \mathrm{BMI}<25 \mathrm{~kg} / \mathrm{m}^{2}$ and $\mathrm{BMI} \geqq 25 \mathrm{~kg} / \mathrm{m}^{2}$ groups. However, no significant associations were found in the $\mathrm{BMI}<18.5 \mathrm{~kg} / \mathrm{m}^{2}$ group. With a trend the same as tSNP rs12951053, one study has shown that SNP rs1042522 in TP53 may be associated with susceptibility to breast cancer among Iranian women with high BMI. ${ }^{14}$

In TP53, compared with the common homozygote A/A of tSNP rs12951053, patients with the heterozygote $\mathrm{C} / \mathrm{A}$ had a risk 1.50-fold higher than triple-negative breast cancer, and displayed a positive association with stage II breast cancer and lymph node-negative breast cancer. However, a study of Jordanian women did not find a significant association between SNP rs12951053 and breast cancer subtype, tumor stage or lymph node status. $^{15}$ Most of the studies on TP53 focus on rs1042522 (Codon 72 Polymorphism), while few on rs12951053 (which is located in intron 7, and the mechanism is not clear). ${ }^{16,17}$ And the rs12951053 also was found associated with invasive serous ovarian cancer risk (median $\left.\mathrm{OR}_{\text {per allele }}=1.65 ; 95 \% \mathrm{CI}=1.20-2.26\right) .{ }^{18}$

In the present study, three tSNPs in NBS1 (rs1805812, rs2735385 and rs6999227 with a heterozygous genotype, uncommon homozygous genotype or individual allele) showed in each case a negative association with luminal $\mathrm{B}$ and triple-negative breast cancer, compared with the common homozygote. However, we did not find any significant association with luminal A and HER2+/HR- breast cancer. In stage II breast cancer, we also found that the three tSNPs displayed a negative association compared with the common homozygote. The tSNPa rs2735385 and rs6999227 also exhibited a negative association with stage III breast cancer. The majority of the three tSNPs displayed a negative association with lymph node status.

In BRIP1, we found that the heterozygous genotype G/ A of tSNP rs7220719 displayed a negative association with luminal A breast cancer and the uncommon homozygote A/A exhibited a positive association with triplenegative breast cancer. However, we did not find any significant association between tSNP rs16945628 and breast cancer molecular subtype, TNM staging or lymph node status. In addition, heterozygous genotype G/A of tSNP rs7220719 also displayed a negative association with stage II breast cancer and lymph node-negative breast cancer.

In tSNP rs2299941 in PTEN, we found that the single allele $\mathrm{G}$ displayed a negative association with each breast cancer molecular subtype, in comparison with the A allele. The heterozygous genotype G/A or uncommon homozygous genotype $\mathrm{G} / \mathrm{G}$ also displayed a negative association with each breast cancer molecular subtype, except triple-negative breast cancer, in comparison with the common homozygote A/A. The same trend was observed with lymph node status. We also found that the heterozygote genotype G/A or uncommon homozygous genotype $\mathrm{G} / \mathrm{G}$ displayed a negative association with stage II or III breast cancer.

The four genes detailed above are directly or indirectly involved in the monoubiquitinated FANCD2-DNA damagerepair pathway and have been found to be significantly associated with the risk of breast cancer. ${ }^{19}$ A previous study of Chinese patients with familial breast/ovarian cancer found that 135 patients were found to carry pathogenic or likely pathogenic mutations (28.1\%), corresponding to 12 different cancer predisposition genes $(8.5 \%$ on non-BRCA1/2 genes, mainly in ATM, CHEK2, PALB2, NBS1, and BRIP1 genes, all of which participate in the homologous recombinant repair pathway). Mutation rates of BRCA1 and BRCA2 have been shown to be higher in triple-negative breast cancer (TNBC) patients than in the non-TNBC group. ${ }^{20}$ The majority of previous reports suggest that genetic polymorphisms may influence the pathological subtype of breast cancer only in ER-positive or -negative breast cancer. As in one study, compared to breast tumours in Caucasian women, they show a higher prevalence of TP53 somatic mutations in ER+ Asian breast tumours, and the presence of TP53 somatic mutations is associated with poorer survival in ER+ tumours. ${ }^{21}$ However, there is no prognostic analysis in this article. Therefore, it is difficult to compare the results of the present study with previous research.

\section{Conclusions}

In this hospital-based case-control study of associations between seven tSNPs of four genes (TP53, NBS1, BRIP1 and PTEN) and risk of breast cancer defined by tumor pathological characteristics and BMI, we found that the majority of tSNPs displayed a negative association with breast cancer and only a few tSNPs exhibited an increased risk of breast cancer (such as rs12951053 in TP53 and rs7220719 in BRIP1, which displayed an increased risk of 
triple-negative breast cancer, and rs12951053 of TP53 and rs16945628 of BRIP1, which displayed an increased risk of breast cancer in the BMI $\geqq 25 \mathrm{~kg} / \mathrm{m}^{2}$ group). Most tSNPs displayed an association with triple-negative breast cancer excepted the tSNP rs2299941 located in PTEN and tSNP rs16945628 located in BRIP1. And the majority of tSNPs displayed a negative association with stage II or III breast cancer. But only one tSNP rs12951053 in TP53 displayed an increased risk of stage II and lymph nodenegative breast cancer. However, the small sample size of the study represents a limitation. Larger and multicenter national studies are required to verify these findings and further exploration of the function of these genes is also required.

\section{Acknowledgments}

The authors thank the Centre for Human Genetics Research, Shanghai Genesky Bio-Tech Co, Ltd for their excellent technical assistance with genotyping analysis. The authors thank all professors, doctors, and nurses in the breast surgery department of Xiangya Hospital for the collection of patient information. The authors thank Associate Professor Guo Wang at the Institute of Clinical Pharmacology, Central South University, for assistance. The authors also thank Associate Professor Xing-Li Li at the School of Public Health, Central South University, for assistance with statistical analysis.

\section{Funding}

This study was supported by grants from the China Hunan Provincial Science and Technology Department (2010TP4053) and the National Natural Science Foundation of China (81001179).

\section{Disclosure}

The authors declare no potential conflicts of interest with respect to the research, authorship, and/or publication of this article.

\section{References}

1. Zheng RS, Sun KX, Zhang SW, et al. Report of cancer epidemiology in China, 2015. Zhonghua Zhong Liu Za Zhi. 2019;41(1):19-28. doi:10.3760/cma.j.issn.0253-3766.2019.01.005

2. Siegel RL, Miller KD, Jemal A. Cancer statistics, 2020. CA Cancer J Clin. 2020;70(1):7-30. doi:10.3322/caac.21590

3. Couch FJ, Hart SN, Sharma P, et al. Inherited mutations in 17 breast cancer susceptibility genes among a large triple-negative breast cancer cohort unselected for family history of breast cancer. J Clin Oncol. 2015;33(4):304-311. doi:10.1200/JCO.2014.57.1414
4. Robson M, Im SA, Senkus E, et al. Olaparib for metastatic breast cancer in patients with a germline BRCA mutation. $N$ Engl J Med. 2017;377(6):523-533. doi:10.1056/NEJMoa1706450

5. Han W, Kang D, Park IA, et al. Associations between breast cancer susceptibility gene polymorphisms and clinicopathological features. Clin Cancer Res. 2004;10:124-130. doi:10.1158/1078-0432.CCR0834-3

6. Garcia-Closas M, Hall P, Nevanlinna H, et al. Heterogeneity of breast cancer associations with five susceptibility loci by clinical and pathological characteristics. PLoS Genet. 2008;4(4):e1000054. doi:10.1371/journal.pgen.1000054

7. Slattery ML, John EM, Stern MC, et al. Associations with growth factor genes (FGF1, FGF2, PDGFB, FGFR2, NRG2, EGF, ERBB2) with breast cancer risk and survival: the breast cancer health disparities study. Breast Cancer Res Treat. 2013;140(3):587-601. doi:10.1007/s10549-013-2644-5

8. O'Brien KM, Cole SR, Engel LS, et al. Breast cancer subtypes and previously established genetic risk factors: a bayesian approach. Cancer Epidemiol Biomarkers Prev. 2014;23(1):84-97. doi:10.1158/ 1055-9965.EPI-13-0463

9. Chen FY, Wang H, Li H, et al. Association of single-nucleotide polymorphisms in monoubiquitinated FANCD2-DNA damage repair pathway genes with breast cancer in the Chinese population. Technol Cancer Res Treat. 2018;17:1-11. doi: $10.1177 / 1533033818819841$

10. Tang LL, Chen FY, Wang H, et al. Haplotype analysis of eight genes of the monoubiquitinated FANCD2-DNA damage-repair pathway in breast cancer patients. Cancer Epidemiol. 2013;37(3):311-317. doi:10.1016/j.canep.2012.12.010

11. WHO Expert Consultation. Appropriate body-mass index for Asian populations and its implications for policy and intervention strategies. Lancet. 2004;363:157-163. doi:10.1016/S0140-6736(03)15268-3

12. Goldhirsch A, Winer EP, Coates AS, et al.; Panel members. Personalizing the treatment of women with early breast cancer: highlights of the St Gallen International Expert Consensus on the primary therapy of early breast cancer 2013. Ann Oncol. 2013;24 (9):2206-2223. doi:10.1093/annonc/mdt303

13. Chen FY, Ou HY, Wang SM, Wu YH, Yan GJ, Tang LL. Associations between body mass index and molecular subtypes as well as other clinical characteristics of breast cancer in Chinese women. Ther Clin Risk Manag. 2013;9:131-137. doi:10.2147/TCRM.S41203

14. Anoushirvani AA, Aghabozorgi R, Ahmadi A, et al. Association of rs1042522 SNP with clinicopathologic factors of breast cancer patients in the Markazi Province of Iran. Open Access Maced J Med Sci. 2018;6(12):2277-2282. doi:10.3889/oamjms.2018.486

15. Al-Eitan LN, Rababa'h DM, Alghamdi MA, Khasawneh RH. Correlation between candidate single nucleotide variants and several clinicopathological risk factors related to breast cancer in Jordanian women: a genotype-phenotype study. $J$ Cancer. 2019;10 (19):4647-4654. doi:10.7150/jca.33857

16. Hao W, Xu X, Shi H, Zhang C, Chen X. No association of TP53 codon 72 and intron 3 16-bp duplication polymorphisms with breast cancer risk in Chinese Han women: new evidence from a population-based case-control investigation. Eur $J$ Med Res. 2018;23(1):47. doi:10.1186/s40001-018-0345-6

17. Afzaljavan F, Chaeichi Tehrani N, Rivandi M, et al. The dilemma of TP53 codon 72 polymorphism (rs1042522) and breast cancer risk: a case-control study and meta-analysis in the Iranian population. Cell J. 2020;22(2):185-192. doi:10.22074/cellj.2020. 6458

18. Schildkraut JM, Iversen ES, Wilson MA, et al. Association between DNA damage response and repair genes and risk of invasive serous ovarian cancer. PLoS One. 2010;5(4):e10061. doi:10.1371/journal. pone.0010061

19. Walsh T, King MC. Ten genes for inherited breast cancer. Cancer Cell. 2007;11(2):103-105. doi:10.1016/j.ccr.2007.01.010 
20. Wang J, Li W, Shi Y, et al. Germline mutation landscape of Chinese patients with familial breast/ovarian cancer in a panel of 22 susceptibility genes. Cancer Med. 2019;8(5):2074-2084. doi:10.1002/ cam4.2093
21. Pan JW, Zabidi MMA, Ng PS, et al. The molecular landscape of Asian breast cancers reveals clinically relevant population-specific differences. Nat Commun. 2020;11(1):6433. doi:10.1038/s41467020-20173-5

\section{Publish your work in this journal}

Clinical Epidemiology is an international, peer-reviewed, open access, online journal focusing on disease and drug epidemiology, identification of risk factors and screening procedures to develop optimal preventative initiatives and programs. Specific topics include: diagnosis, prognosis, treatment, screening, prevention, risk factor modification,

Submit your manuscript here: https://www.dovepress.com/clinical-epidemiology-journal systematic reviews, risk \& safety of medical interventions, epidemiology \& biostatistical methods, and evaluation of guidelines, translational medicine, health policies \& economic evaluations. The manuscript management system is completely online and includes a very quick and fair peer-review system, which is all easy to use. 